\title{
IUMRS-ICAM-2009 Explores Advanced Materials from Research to Innovations
}

"In Brazil, everywhere I go there is a soccer field and everyone is playing soccer," a friend once commented to Sergio Rezende, Minister of Science and Technology in Brazil, in an effort to explain why Brazilians excel in the sport. In contrast, Rezende remarked that because there has not been a culture surrounding science in his country, few people are attracted to it. However, the Brazilian government is changing that by trying to make science as pervasive as soccer, Rezende said in his opening plenary address at the International Conference on Advanced Materials 2009 (ICAM 2009) organized by SBPMat, the Brazilian Materials Research Society, in Rio de Janeiro on September 20-25, 2009. The ICAM conferences, conducted within the umbrella of the International Union of Materials Research Societies (IUMRS), are held every other year, and this is the 11th in the series and the first held in Brazil.

The scientific and technical program, organized by Guillermo Solórzano of PUC-Rio (Chair of ICAM 2009) and Elisa Baggio Saitovitch of CBPF-Rio (Program Chair), presented a range of topics at the frontiers of materials research, technology, and engineering. With 1560 attendees (45\% students) from 60 countries, significant opportunities were available for materials researchers around the world to meet and form collaborations.

Rezende, a materials scientist himself who co-organized the first meeting in Brazil on materials 40 years ago, elucidated on Brazil's Action Plan 2007-2010, the government's effort to stimulate science, technology, and innovation. He stated that the groundwork had been laid for this effort due to the country's currently favorable macroeconomic conditions, citing low inflation, low external vulnerability, a sound fiscal policy, a strong banking sector, and a large domestic market. Additionally, he said, the industrial sector has become more aware of the need for interaction between research institutions and industry, and the scientific community has matured. Among the areas emphasized in the action plan for research and development are biotechnology, nanotechnology, health, and energy.

While federal funding totaling USD $\$ 26$ billion for this action plan came from a variety of sources, half of this amount was received directly from the Ministry of Science and Technology. Rezende showed that since the plan's release in 2007, the number of federal scholarships has risen significantly, the number of federal universities has increased dramatically from 43 in 2002 to 230 in 2009 with a more uniform distribution throughout Brazil, and the number of scientific publications has increased by a factor of 16 as compared to a world production increase of only 3.5. These statistics demonstrate the rapidly increasing presence of the Brazilian scientific community.

\section{Energy Issues Covered in Forum and Technical Sessions \\ "Guys, where did you hide the fuel} cells?" posed Enrico Traversa, looking at new energy policies in the United States and around the world. While fuel cells alone cannot solve the world energy challenges, said Traversa, who is from the International Center for Materials Nanoarchitectonics (MANA), National Institute for Materials Science (NIMS), Tsukuba, Japan, they are an important component in integrating different technologies and bridging the current portfolio of fossil fuels to a more sustainable energy portfolio. Often, fuel cells are tied to hydrogen, but fuel cells can operate with hydrocarbons, methanol, ethanol, and other renewable sources as well. Although if hydrogen is used, emission is water clean enough to drink, Traversa said.

During the Energy Forum organized by IUMRS President Howard Katz (Johns Hopkins Univ., USA), Traversa was not only expounding on the benefits of fuel cells, but addressing a key theme running through ICAM-2009: The energy situation is a global problem requiring the attention of governments and researchers from around world. Through the Energy Forum and numerous technical sessions during the week, researchers described government policies, materials challenges, and technical results of their portion of the puzzle.

The U.S. Department of Energy's strategic plan addresses the need for energy diversity, reducing environmental impacts of energy, creating more flexible and more reliable energy infrastructure to handle increasing capacity, and improving efficiency economically, said Julia M. Phillips, director of the Physical, Chemical, and Nano Sciences Center at Sandia National Laboratories. In her presentation at the Energy Forum, Phillips said other governments have similar goals, although with some variations in emphasis. Brazil has a focus on energy diversity and self- sufficiency, Europe includes a focus on international relations, and Japan includes increasing the role of market principles. Securing a viable energy future for humankind will require development of carbon-neutral energy sources, including renewable sources, and improvements in energy efficiency, Phillips said.

As an example, Phillips focused on highefficiency, cost-effective solid-state lighting. For high-performance and affordable white light, she said, there are challenges at every stage. First, efficient injection and transport of charge is needed, including higher $p$-type charge carrier concentrations, mobilities, and contacts. Next, a better understanding is needed of radiative and nonradiative recombination. The next major challenge is phosphors for color conversion and mixing. Fluorescence lighting phosphors are unsuitable and red needs significant efficiency improvements. Finally, she said, light extraction efficiency needs to be improved, as photons get stuck in the layer with high index of refraction. Nanoscale photonic crystals can help address this challenge, Phillips said.

Shefford Baker (Cornell University, USA, and 2009 president of the Materials Research Society) introduced the topic of renewable energy. Speaking on behalf of David Ginley (National Renewable Energy Laboratory, USA, and vice president of MRS), Baker said that for renewable energy to make an impact on the terawatt scale, production of energy generation technologies on an unprecedented scale and rate will be required. While renewable energy such as wind and solar is growing rapidly, that rate of growth needs to continue just to grow the $13 \%$ worldwide renewable energy slice to $14 \%$ (and $6 \%$ to $9 \%$ in the United States) from 2006 to 2030. This is because the worldwide energy consumption pie is projected to grow by $55 \%$ in that time.

Solar has much less installed capacity and higher costs than wind, Baker said. The United States has about 1 GW of installed capacity of photovoltaics (PVs) and $420 \mathrm{MW}$ of concentrating solar power, with current rates of $18-23 \notin / \mathrm{kWh}$ and $12 \phi / \mathrm{kWh}$, respectively, with potential for 5-10 $/ \mathrm{kWh}$ and $6 \not / \mathrm{kWh}$, respectively, by 2015 . Grid parity has been met in some places such as California and Italy, Baker said. The PV portfolio ranges from low-cost, low-efficiency organics to high-efficiency, high-cost multijunction concentrators, with crystalline silicon in 
between. The wide range of options and materials challenges creates a competitive playing field, Baker said.

In the biofuels area, Fernando Galembeck, from the University of Campinas, Brazil, said that Brazil is currently producing 180,000 barrels of ethanol per day from sugarcane, replacing half of the gasoline in the country. Additionally 3\% of electricity comes from bagasse-the fibrous residue left after juice extraction-and chemicals for food and surfactants fill another use of sugarcane other than for food. Currently $1 \%$ of available arable land is used for sugarcane for ethanol, with more than 20 times that amount still available for use, taking into account the preservation of areas such as the Amazon, Pantanal, and the Mata Atlântica. Brazil is not alone in its potential for biofuel production, however, with a swath of tropical latitudes around the globe from Africa to Australia showing potential, Galembeck said.

Experimental units in the state of São Paulo are focused on another native plant, manioc (also known as yucca or cassava), and have achieved a $25 \%$ reduction in energy consumption using new machinery. Beyond ethanol, higher energy density can be achieved through biodiesel, and this sector is likely to grow after a leveling off of ethanol production. Among the challenges, more work is needed to conserve fertilizer, of which $50 \%$ goes to pollution. Finding a way to use the nitrate and ammonia-rich effluents as raw materials might be a route to pursue, Galembeck said, and reducing evaporation is another need.

Overviews of the global response to energy challenges were also delivered in some of the technical sessions. C. English (National Nuclear Laboratory, UK) opened his talk in Symposium $\mathrm{N}$ by highlighting the recent renaissance in the United Kingdom in the field of nuclear-related materials research. Whereas the availability of nuclear expertise had been in steady decline in the UK for many years, English said that a recent shift in the country's energy policy resulted in nuclear power emerging as a key source due to the security and diversity of its supply and its low carbon footprint. Increased interest in nuclear materials research and development (R\&D) in industry and academia has also benefited from significant advances in specimen preparation techniques, microstructural characterization, and modeling. Current nuclear materials research in the UK focuses upon advanced gas-cooled reactor life extension, materials problems associated with decommissioning and waste management, and light water reactor research including reactor pressure vessel embrittlement, environment-assisted cracking and stress corrosion cracking, and the performance of $\mathrm{Zr}$ alloys, a material often employed in nuclear facilities.

A fundamental understanding of the fracture deformation mechanisms in zirconium alloys is of prime importance for nuclear safety. M. Ruda of the National Atomic Energy Commission in Argentina delivered a presentation in Symposium Z explaining that in addition to the presence of twinning dislocations (which were observed in all cases), the phase transformations occurring at the crack tip for certain geometric orientations play an extremely important role in improving the ductility of this material. By employing atomistic static simulations of mode I fractures using empirical embedded atom potentials to simulate equilibrium quasistatic crack growth, Ruda found that crack tips advanced much more quickly and required much less energy for cracks lying in the prismatic plane. When the crack lay in the basal plane, however, a martensitic hcp to bcc phase transformation near the crack tip resulted in increased ductility. In the pyramidal case, where the highest amount of energy was required for crack propagation and crack tip blunting was most pronounced, both the high temperature bcc and the high pressure phases were observed.

Among other energy-related studies reported at the conference, A. Khan (Univ. of South Carolina, USA) presented his work, in Symposium M, on advancing processing for light-emitting diodes (LEDs) operating below $300 \mathrm{~nm}$. To shift operating wavelengths to the UV, high concentrations of $\mathrm{Al}$ are needed to dope the GaN and InGaN layers, but increasing Al content causes cracking and other defects during growth. By using a pulsed deposition method, where mass sources are alternated during growth, cracking and dislocations could be limited because $\mathrm{Al}$ mobility is increased. Also, hierarchical heterostructures were built to mitigate heteroepitaxial strains. Instead of using graded structures between layers, Khan placed thinner individual alternating layers of $\mathrm{GaN}$ and InGaN as the interlayer. Using these methods, defect concentration was improved by an order of magnitude over other similar devices, but the UV LEDs still have 10-20 times higher defect concentrations than visible light LEDs.

Most polymer solar cells research concentrates on increasing electron production in photosensitive polymers, but J. Durrant (Imperial College, UK) has been researching electron-hole recombination, which he believes has been limiting polymer solar cell efficiency. The electrostatic attraction within the electron-hole pair often results
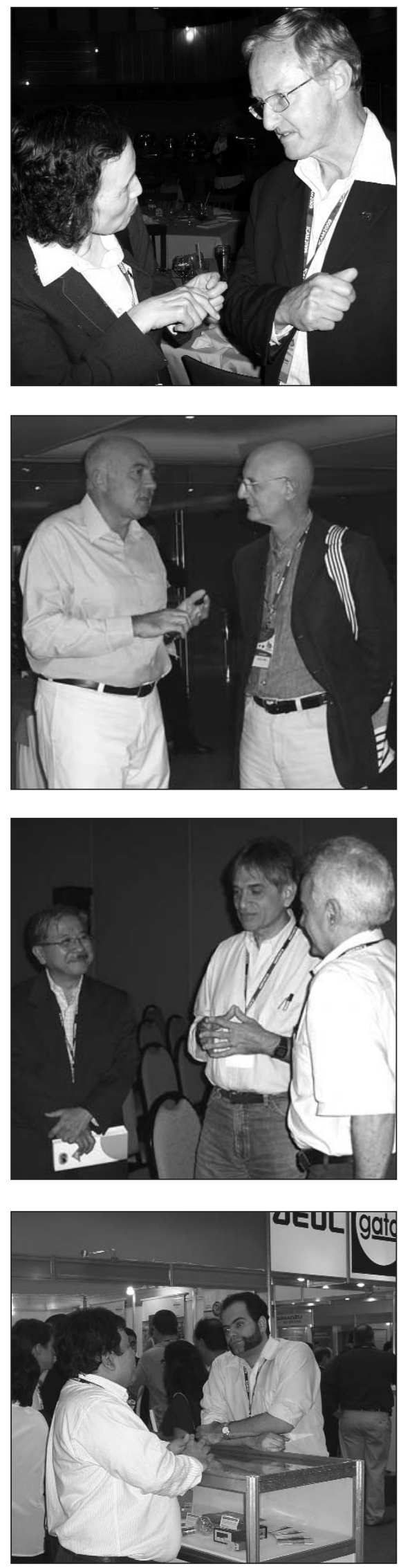
in recombination rather than conduction, said Durrant in Symposium M. He has found that polymer crystallinity, the lengths of alkyl side chains, and high cell biases do not substantially affect recombination rates, but increasing the energy difference between the polymer donor and the fullerene acceptor seems to decrease recombination rates.

Metal alloy hydrides show promise for hydrogen storage applications if hydrogenation can be accelerated, said J. Huot (Université du Québec à Trois-Rivières, Canada). The use of severe plastic deformation (SPD), a process that creates defects and stores energy within them, could provide the means to achieve this desired hydrogenation acceleration. Huot's team set out to determine whether cold rolling, a well known low-energy SPD method, could compete with ball milling, a highenergy process unsuitable for scale up. Huot reported in Symposium V that cold rolling $\mathrm{Mg}$ with $2.5 \mathrm{at} \% \mathrm{Pd}$ does indeed lead to materials that are easier to activate and that provide better resistance to air than their ball-milled counterparts. However, the cost of Pd prevents this particular approach from being scaled up. Huot also reported that challenges remain in understanding the contribution of composition, microstructure, and defects to the resulting properties.

In Symposium $\mathrm{O}$ on direct energy conversion systems, R. Funahashi (Japan Natl. Inst. of Advanced Industrial S\&T) discussed his team's efforts in converting waste heat to electricity by using thermoelectric oxides as an alternative to thermoelectric semiconductors. Funahashi discussed process and reliability improvements in $\mathrm{Ca}_{3} \mathrm{Co}_{4} \mathrm{O}_{9}$ ( $p$-type)$\mathrm{CaMnO}_{3}$ (n-type) thermoelectric oxide systems where sintering was used to produce polycrystalline oxides that were then sectioned and fabricated into thermoelectric devices. During thermal cycling, resistance increased in the $n$-type material, which Funahashi mitigated by moving to a spray dried powder. He also showed that contact resistance between $p$-type oxide and the metal electrode could be decreased by mixing ceramic powder with commercially available Ag paste prior to application. Funahashi left his audience with a vision of increasing thermoelectric conversion efficiency to the point where even the smallest heat-producing devices (e.g., cell phone batteries) could be harnessed to yield appreciable thermoelectricity.

In Symposium S, P. Bristowe (Cambridge Univ., UK) described his work on lowemissivity architectural glasses to reduce heat loss in modern buildings. Multilayer thin films are currently used to limit infrared transmission in low-emissivity glasses, but these films slowly degrade over time due to failure mechanisms in the interface-occupied by a half monolayer of $\mathrm{H}$ atoms-between $\mathrm{ZnO}$ and $\mathrm{Ag}$ films. Through first principle calculations and simulation, Bristowe determined that the $\mathrm{H}$ atoms were bonded to the oxygen atoms and shared chemical bonds with the Ag monolayer and that high hydrogen contamination areas nucleate delamination in real systems. He further used density-functional-theory-based first principles calculations to show that $\mathrm{Al}^{3+}$ preferentially segregates to the $\mathrm{Ag} / \mathrm{ZnO}$ interface and charge compensates for the terminating oxygen layer. Hydrogen adsorption is dramatically reduced in doped samples and the ideal work of separation increased three times over that in undoped materials.

To improve carbon capture processes, J. da Costa (Univ. of Queensland) presented gas separation technologies based on porous silica membranes for use in gasification systems. The membranes separate gases by molecular sieve action where selectivity is determined by pore size and distribution. Da Costa developed techniques to synthesize and characterize membranes with tailored pore size, morphology, and distribution. He found that selectivity could be tailored for a wide range of gases including $\mathrm{H}_{2}$, $\mathrm{He}, \mathrm{N}_{2}, \mathrm{CO}, \mathrm{CO}_{2}$, and $\mathrm{CH}_{4}$. The primary technical hurdle for this technology is increasing mechanical strength of the membranes.

Plenary speaker Jacques Amouroux (Université Pierre et Marie Curie, France) gave a different take on $\mathrm{CO}_{2}$, exploring the possibilities of transforming it into fuel as a way of storing energy to be used later. By combining $\mathrm{CO}_{2}$ from fossil fuel burning (and other processes) with hydrogen created through renewable and non- $\mathrm{CO}_{2}$-producing routes, fuels can be produced such as syngas, methane, methanol, and other hydrocarbons. Pilot plants are already in operation or under development such as for Synfuel (Canada, USA), methanol (China, USA), Synfuel through Fisher Tropsch (gasification of coal and waste) (South Africa, USA), and Syngas from a high power arc plasma (Russia, USA). Amouroux challenged the international materials community to develop new catalysts, efficient methods for solar water splitting, and economic evaluation of these processes.

\section{Nanomaterials Bring Biomedical Research Closer to Treatment}

Nanoparticles are of great significance for biomedical applications because they are on the same size scale as biological entities, said L. Lagae (IMEC, Leuven, Belgium). In her work on tracking stem cells in vivo through MRI imaging, Lagae described the need for monodisperse magnetic nanoparticles (NPs). Her group synthesized magnetic nanoparticles of

\section{Materials Research Utilizes Natural Fibers of Brazil}

Agribusiness in Brazil employs 37\% of the total working population, represents $42 \%$ of exports, and accounts for $33.8 \%$ of the gross national product. The Brazilian Agricultural Research Corporation, Embrapa, for example, employs 9,000 people including 2,200 researchers, and has a budget of $\$ 700$ million/year. L.H.C. Mattoso of Embrapa said that the ministry is currently developing agro-based materials using nanotechnology. Innovations at Embrapa include an electronic tongue for analyzing wines, hydrogels using methyl cellulose, and nanoparticles of natural polymers.

In a collaborative project between Embrapa and the U.S. Department of Agriculture, researchers are examining ways to improve quality and shelf life of food while reducing disposable packaging. Mattoso described how thin, edible films that can be wrapped around food, keeping flavor and moisture in with a minimum of waste, can be strengthened with cellulose nanofibers and chitosan nanoparticles. Cellulose fibers can be derived from a range of sources in Brazil, including cotton, sisal, sugarcane bagasse, corn straw, pineapple, and nanocellulose from coconut waste. Nanowhiskers from coconuts have various shapes and sizes, typically ranging 100-500 $\mathrm{nm}$ in length and $4-6 \mathrm{~nm}$ in diameter. The fibers are bleached to remove the lignin and then self-assembled to build up multilayered films. Incorporation of chitosan nanoparticles in the films improved their mechanical and barrier properties significantly, Mattoso said. The nanoparticles tend to occupy the empty spaces in the pores of the HPMC matrix, improving film tensile strength and barrier properties, Mattoso said. Cellulose nanofibers also improved mechanical and barrier properties of mango puree edible films, doubling the tensile strength and nearly halving the water permeability by adding 36 grams of fibers per 100 grams of mango puree. 
$\mathrm{Fe}_{3} \mathrm{O}_{4}, \mathrm{CoFe}_{2} \mathrm{O}_{4}$, and $\mathrm{MnFe}_{2} \mathrm{O}_{4}$ using a thermal decomposition method. The particles required a surface coating for use in biological samples. Lagae described the effects of material composition, size, and coating on the MRI contrast. Stem cell therapy approaches are being considered for malignant glioma in the brain, as an example of an application.

P.C. Morais (Univ. of Brasilia) described his group's work with packaging NPs with drugs in lyposomes. The lyposomes prevent the drugs from being attacked by the body but can be destroyed by vibrating the nanoparticles with an external ac field. Using this method, doctors can directly control drug release after it is introduced into the body. Morais also showed that magnetic nanoparticles could be injected into tumors and heated with an applied magnetic field for hypothermic therapy. His materials are currently in Phase I human testing.

E. Traversa (Natl. Inst. for Materials Science, Japan) used adult stem cells for his research addressing cardiac regenerative medicine. Synthetic and/or natural materials can guide stem cells to organize into complex architectures that mimic native tissues. While discussing various strategies undertaken to develop optimal three-dimensional (3D) scaffolds, Traversa said that "hierarchical porosities are needed in a single scaffold at the millimeter scale to help nutrition and vascularization, at the micrometer scale to accommodate cells, and at the nanometer scale to favor the expression of extra-cellular matrix components." His group selected low-cost and user-friendly processing techniques such as phase separation and electrospinning to fabricate tunable, hierarchically porous 3D matrices that mimic aspects of a cell's microenvironment. Scaffolds made from the directional thermally induced phase separation (dTIPS) technique and electrospinning gave them better control over the desirable scaffold properties. Scaffolds made of poly(L-lactic acid) (PLLA) with an overall porosity of $93 \%$, prepared by dTIPS, showed the ability to grow 1-mm thick, healthy tissues after 14 days using in vitro cell culture experiments with mesenchymal stem cells, exploiting the highly porous microstructure of the engineered scaffolds.

Inspired by the need to generate functional artificial tissues for transplants, A.K. Khademhosseini (joint Harvard-MIT Health Sciences and Technology) mimicked the cell's microenvironment and generated 3D tissues by merging microengineering technologies with hydrogel biomaterials. His research group has made seminal contributions by using bottom-up approaches to generate tissues by the assembly of shape-controlled cell-laden microgels (i.e., tissue building blocks) that resemble functional tissues. In this approach, microgels were fabricated and seeded with different cell types and induced to self assemble, to generate 3D tissue structures with controlled microarchitecture and cell-cell interactions.

Plenary speaker S. Suresh (Massachusetts Inst. of Technology, USA) integrates the knowledge of materials science with cellular biology, biochemistry, genetics, and mechanics to understand the mechanistic origins of diseases such as malaria. His work has shown that the biophysical characteristics of cells and subcellular components change drastically with the onset of malaria parasitization. For instance, red blood cells infected with malaria become stiff and sticky. These infected cells do not move easily through small blood vessels. Suresh said that once invaded by malaria parasite, biochemical and biomechanical mechanisms of human red blood cells change within 48 hours. Suresh used 3D dissipative particle dynamics to simulate the flow of human RBCs through microvasculature (blood vessels and spleen), which was experimentally accomplished by in vitro microfluidic experiments. His group demonstrated a microfluidic device that has the ability to differentiate between healthy and diseased RBCs; such a device could help in the detection of malaria.

Plenary speaker S.S.P. Parkin (IBMStanford SpinApps, USA), well known for inventing the spin valve, has entered the realm of brain circuitry, or "a brain in a box." He discussed his efforts to bring 3D architectures based on biological neurons and synapses to logic devices. Parkin described a device wherein a magnetic tunnel junction is sandwiched between two conducting wires. When the wires are placed perpendicular to each other, the conductivity of the tunnel junction can be controlled by electronic pulses across the wires. By varying the order, spacing, and voltage of pulses, the magnetic tunnel junction's electronic properties could be continuously adjusted to both increase and decrease conductance. The ability to modify electronic properties as a function of pulse order and spacing is a fundamental property of synapses causing the junctions to behave similarly to biological systems. If these devices can be integrated into a 3D architecture, Parkin said, they may behave similarly to brain synapses.

\section{Nanoscience and Nanotechnology}

Existing knowledge of the mechanical behavior of nanoscale materials systems is
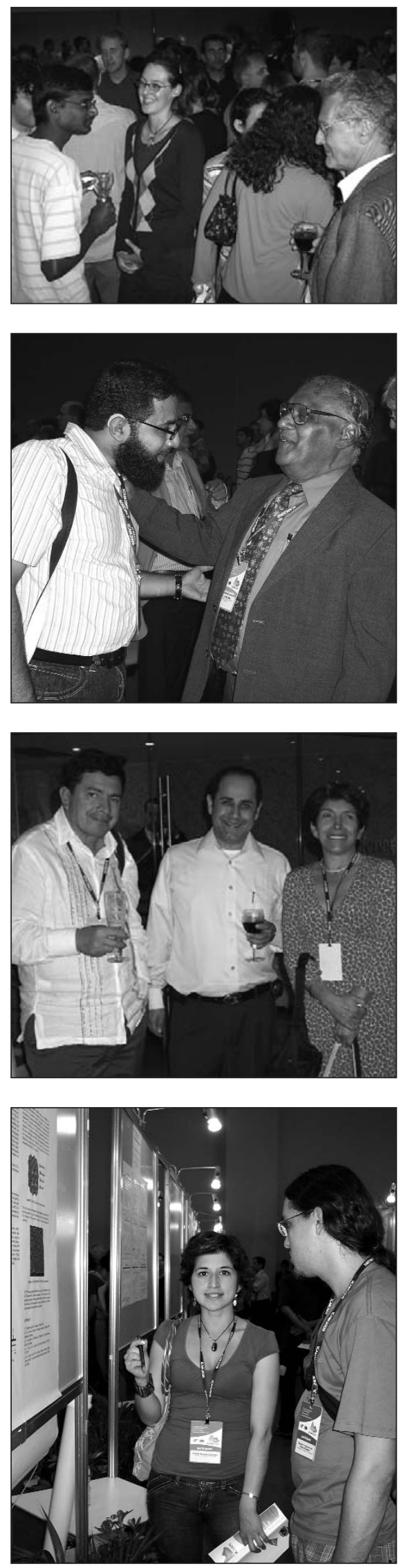
limited to computational simulations and has not been supported by experiments due to the huge differences in applied deformation rates. This aspect makes the underlying physics difficult to understand. Motivated by this challenge, D. Ugarte (Universidade Estadual de CampinasUNICAMP, Brazil), through an experi- mental and theoretical study, addressed the tensile deformation of $\sim 1$-nm-wide metal wires by an atomic resolution timeresolved electron microscopy study and $a b$ initio calculations. Size dependence of the mechanical behavior of nanoscale metal rods was determined by observing the positions and displacements of atoms

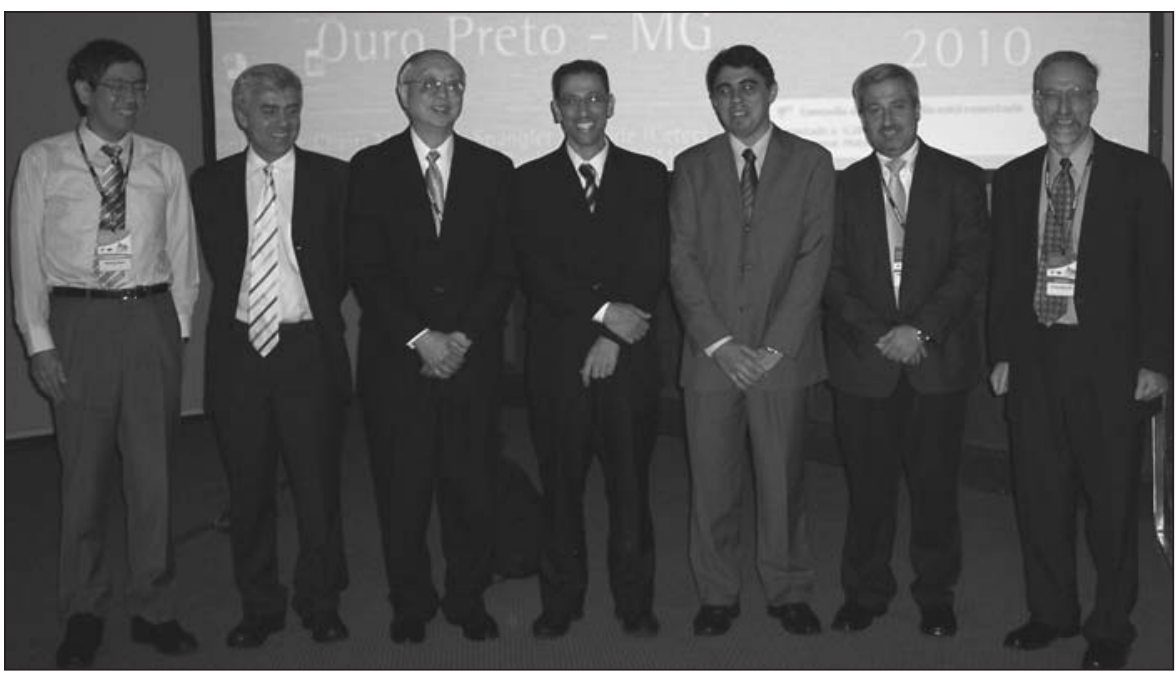

IUMRS President Howard Katz (far right) and IUMRS General Secretary R.P.H. Chang (third from the left) present the $\mathbf{2 0 0 9}$ Sōmiya Award to research team (left to right): Riichio Saito of Tohoku University, Japan; Marcos Pimenta of Universidade Federal de Minas Gerais, Brazil; Ado Jorio of Universidade Federal de Minas Gerais, Brazil; Antonio G. Souza Filho of Universidade Federal do Ceara, Brazil; and Mauricio Terrones of Instituto Potosino de Investigacion Cientifica y Technologica, Mexico. Not shown are team members Mildred S. Dresselhaus of the Massachusetts Institute of Technology, USA and Morinobu Endo of Shinshu University, Japan.

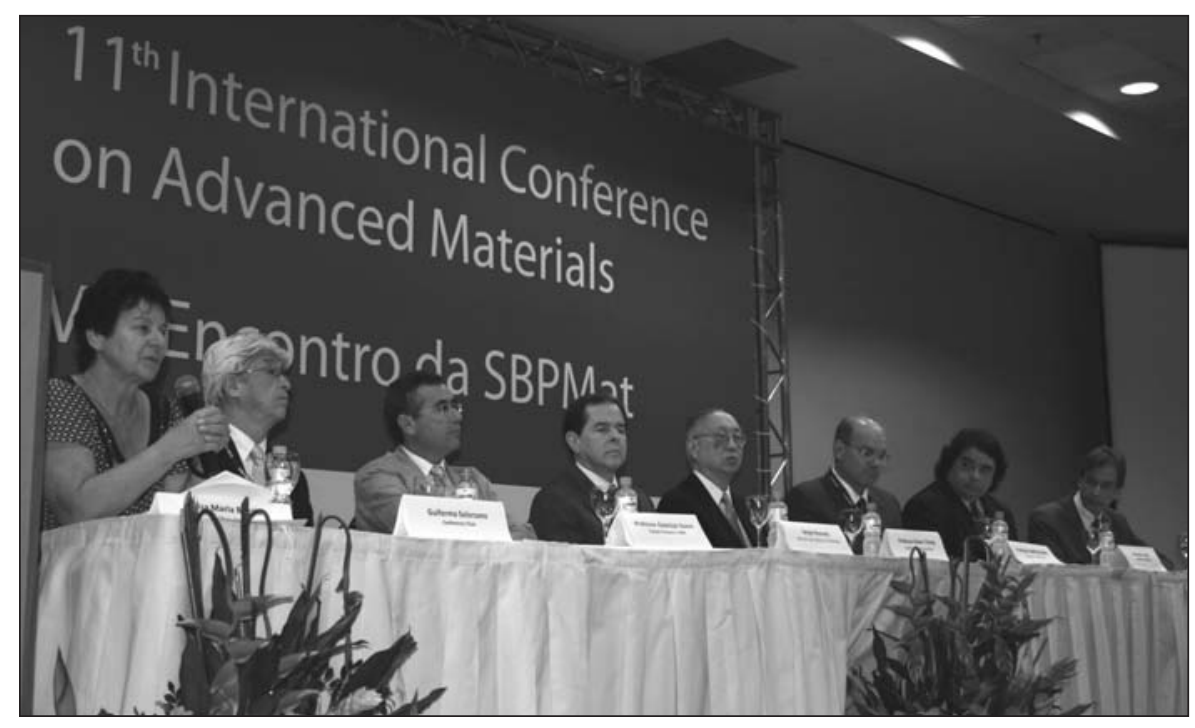

Opening ceremony for IUMRS-ICAM 2009 (left to right): Program chair Elisa Baggio Saitovitch, conference chair Guillermo Solórzano, Abdelilah Slaoui (European-MRS), Brazilian minister for Science and Technology Sergio Rezende, Robert Chang (IUMRS), Shefford Baker (Materials Research Society), Brazilian-MRS (SBPMat) president Fernando Lázaro Freire Jr., and Sandoval Carneiro Jr. (CAPES, Brazil).

during the mechanical stretching of 1-nmwide metal rods at different temperatures. Ugarte's research team did not observe any defects in gold rods deformed at room temperature, while for those deformed at $150 \mathrm{~K}$, stacking faults were generated. The researchers hypothesized that planar defect energy barriers may become so small due to size reduction, and that ambient thermal energy can overcome them. The nanorods displayed an extended elastic regime until a mechanism with a sufficiently high blocking barrier can be nucleated. The researchers verified their model by studying 1-nm rods made of another fcc metal, platinum, which showed different energy barriers in macroscopic systems.

Shape memory alloys (SMAs) are used as actuation materials in microelectromechanical systems (MEMS) and their biocompatibility characteristics also allow them to be used in bioMEMS devices such as microvalves and pumps, which require the ability to move with large force but with smaller frequencies. In her study on nanoindentation response of $\mathrm{NiTi}$ shape memory thin films, A.G. Ramirez (Yale Univ.) found that crystallization of NiTi films occurs over a range of temperatures rather than at a fixed temperature as was previously thought. She is particularly interested in the mechanical properties of these films as a function of the volume crystallized. She carried out shallow penetration nanoindentation experiments on as-deposited and fully crystallized films, which served as guides for her analysis to observe the effects of crystallization on mechanical properties. The films exhibited a bimodal elastic response, that is, softer in crystalline regions and stiffer in amorphous regions. The elastic moduli of the amorphous regions of a semi-crystalline film were seen to be more than that of the as-deposited film, which was attributed to the nanocrystalline regions in the amorphous phase which undergo structural relaxation, making amorphous regions harder than their crystalline counterparts. Ramirez said that these small variations in local material structure can be utilized to make various nanocomposites with varying degrees of crystallization which can be used to modify mechanical properties at the nanoscale.

In polymer-clay nanocomposites (PCNs), interactions between clay nanoparticles and the polymer matrix play a significant role in mechanical property enhancement. S. Sinha (CSIR, South Africa), and his group studied the degree of dispersion in polymer-clay nanocomposites using small angle x-ray scattering (SAXS) and correlated the predicted morphology with the melt morphological 
properties of nanocomposites. They chose poly[(butylenes succinate)-co-adipate] (PBSA) as a model polymer matrix due to its low temperature processability and good thermal stability. Sinha found good agreement between experimentally obtained scattering patterns with theoretically calculated ones. His work demonstrates that SAXS is a useful technique to study the relation between rheology and structure in clay-containing polymer nanocomposites.

\section{Metallic Glasses Expand into New Fields}

Plenary speaker A. Inoue, president of Tohoku University, Japan, discussed bulk glassy alloys. He said applications of $\mathrm{Zr}-$, Ti-, and Fe-systems continue to expand into new fields as useful and interesting properties are being designed and observed. Inoue's group developed guiding rules for forming glassy alloy systems, and subsequently, using these guidelines, a number of glassy nonferrous and ferrous alloy systems have been discovered by various research groups. Within the past year, his group has made glassy alloys of $\mathrm{Zr}-\mathrm{Al}-\mathrm{Co}, \mathrm{Zr}-\mathrm{Al}-\mathrm{Cu}-\mathrm{Fe}$, $\mathrm{Zr}-\mathrm{Al}-\mathrm{Cu}-\mathrm{Co}, \mathrm{Ni}-\mathrm{Pd}-\mathrm{P}-\mathrm{B}-\mathrm{Si}$, and $\mathrm{Fe}-\mathrm{Nb}-$ P-B-Si with specimen diameters of $5 \mathrm{~mm}$ to $25 \mathrm{~mm}$. Stabilization of supercooled metallic liquids have yielded new materials science and technology fields, he said. A number of Fe-based bulk metallic glasses have been developed and commercialized, including Liqualloy and SENNTIX.

\section{Graphene Demonstrates Extraordinary} Electronic Properties

Possessing single-, bi-, or few- $(\leq 10)$ layers of carbon atoms forming six-membered rings, graphene has captured the interest and attention of scientists from various disciplines. Plenary speaker C.N.R. Rao of the Jawaharlal Nehru Centre for Advanced Scientific Research, India, said the extraordinary electronic properties of single- and bi-layer graphene are unique and unexpected. He said that a new technique just recently reported for synthesizing graphene involves an arc discharge of a graphite rod in a $\mathrm{H}_{2}+\mathrm{He}$ environment. Transmission electron microscopy and atomic force microscopy reveal that it has a surface area of $2,600 \mathrm{~m}^{2} / \mathrm{g}$.

With excellent electrochemical properties, graphene can act as a supercapacitor, said Rao. The edges of graphene sheets have non-bonding $\pi$-electrons giving rise to edge states and magnetism in the material. Another recently published study showed that graphene plus a singlewalled carbon nanotube and graphene plus nanodiamond yielded orders of mag- nitude improvement in mechanical properties over those of graphene alone.

\section{Electron Microscopy Capable of \\ Picometer Resolution}

Understanding atomic structure and its effects on physical properties is a primary goal of materials science, but researchers have only recently begun to "see" on the atomic scale. In his plenary address, Knut Urban (Research Center Jülich, Germany) described electron microscopy methods capable of picometer resolution. Urban pioneered aberration-corrected transmission electron microscopy (TEM) in the 1990s, demonstrating the first aberrationcorrected microscope and developing analytical methods for structure determination on the quantum scale.

To demonstrate the utility and resolution of this technique, Urban presented case studies in $\mathrm{YBaCuO}, \mathrm{BaTiO}_{3}$, and lead zirconium titanate (PZT). Urban used his technique to calculate atomic displacements resulting from $90^{\circ}$ tilt boundaries in $\mathrm{YBaCuO}$ where regression analysis allowed 4 pm resolution. In $\mathrm{BaTiO}_{3}$, Urban studied $\Sigma 3\{111\}$ twin boundaries. These types of twins are associated with oxygen deficiency during growth of $\mathrm{BaTiO}_{3}$ films, and atomic resolution was used to prove oxygen deficiency at the twin boundaries. By comparing the intensity amplitude of oxygen planes in the electron "images," Urban showed that only $68 \%$ of oxygen sites were occupied. Furthermore, he calculated bond distances at the twin boundaries and showed that Ti-Ti distance increased while Ba-Ba distance decreased. Urban also showed the effect of a single dislocation during epitaxial growth. He studied PZT films grown on $\mathrm{SrTiO}_{3}$ substrates when dislocations were located near the film interface. He calculated atomic positions and found that dislocations in the $\mathrm{SrTiO}_{3}$ substrates more than $15 \mathrm{~nm}$ from the interface caused a $50 \%$ polarization reduction in the PZT layer. Urban said that this may explain the dead layer effect common to ferroelectric thin films.

\section{Supramolecular Materials See Applications from Sensing to \\ Cancer Therapy}

The supramolecular approach to nanotechnology, said plenary speaker Henrique E. Toma (University of São Paulo, Brazil), is creating molecular building blocks from atoms and molecules, which can then be assembled to form nanostructured materials, allowing for the tailoring of functionality and properties yielding functional materials and devices. Toma said molecular and supramolecular materials can be used for
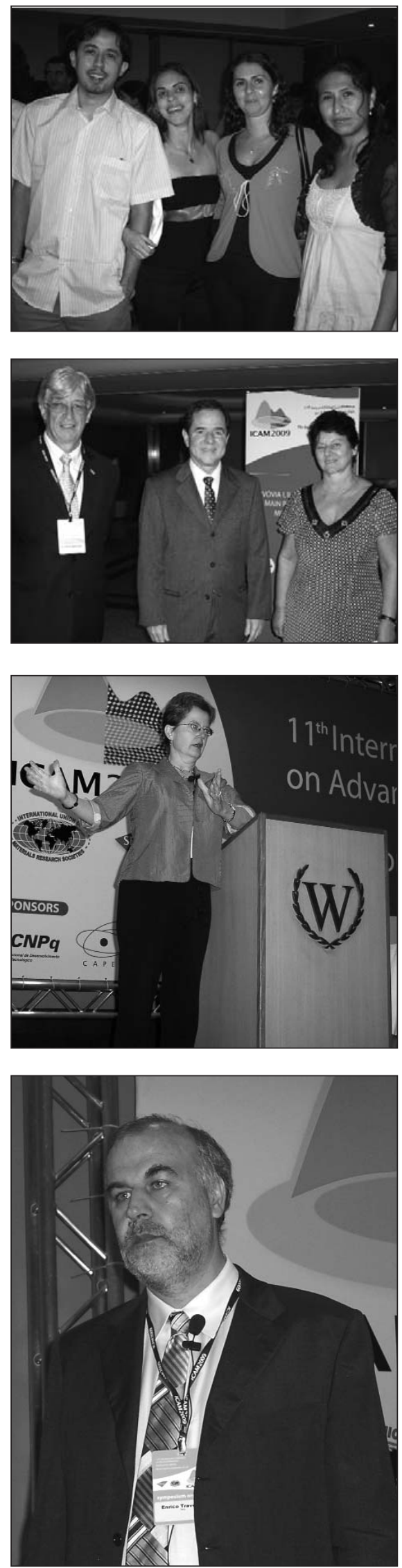
a number of applications in various fields, enabling this area of research with a rich future. Examples cited by Toma include supramolecular chemistry used to form very sensitive and powerful surfaceenhanced Raman spectroscopy (SERS) molecular probes. In one example, gold nanorods combined with antibodies with supramolecular chemistry yielded a way to identify cancer cells using SERS, wherein there was a clear difference in the Raman spectra of healthy and cancerous cells. This was also used in photothermal cancer therapy. In another example, silver nanoparticles were functionalized with imidazoles in poly-2-vinylpyridine electrospun hydrogels for use as bactericidal-fungicidal dressings for treating wounds. Some other examples mentioned by Toma included nanosensors for security, photoelectrochemical cells, superparamagnetic nanoparticles combined with supramolecular chemistry for biocatalysis and for capturing cells, and vanadium oxide-supramolecular porphyrin nanocomposites used as an ethanol fuel sensor.

\section{Materials Education Needs to Address Global Challenges}

The Ouroborus, an ancient symbol depicting a serpent swallowing its own tail and thus forming a circle, can be used to represent the importance of uniting scientific work performed at the microand nanoscale with humankind and the universe as a whole, said S. Mascarenhas (Univ. of São Paulo, São Carlos) in his keynote address on the challenges facing materials education in the 21st century. As the global population is expected to double by the end of the century and as a result of global climate changes, he said there will be an increased demand for energy, water, food, health care, and sustainability. Mascarenhas emphasized the crucial role that nanotechnology, and specifically bionanotechnology, could play in overcoming these challenges through the development of nanomaterials such as catalysts, hydrogen and biomass systems, nano-agro materials, and bionano-pharmaceuticals. In order to tackle these global challenges, efforts are needed to create a new educational structure in materials science and nanotechnology through the implementation of interdisciplinary curricula, continued education, distance learning, and network innovation projects.

Likewise, to overcome scientific illiteracy and misconceptions among the general public, the big topics of today-including climate change, energy resources, nanotechnology, health, and water resourcesshould be used to capture the public's interest, said J.E.E. Baglin (IBM Almaden Research Center, USA). In a society that relies heavily on the use of advanced technologies, a basic knowledge of science is needed to ensure the implementation of intelligent business, government, and legal decisions and to avoid the victimization of the non-technical community in these areas.

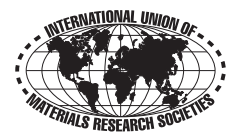

\section{World Materials Summits for Energy Investigate Economically Viable Materials Research Directions}

Recognizing the strategic importance of materials research for the world's energy future, the Materials Research Society (MRS), the European MRS (E-MRS), and the Chinese MRS (C-MRS) are collaborating in a joint endeavour to convene science policymakers across international borders to discuss the role of materials for future energy sustainability. The focal point of these collaborations has been two World Materials Summits for Energy, which were conceived not as just another scientific conference but as an event to bring together global leaders in science, technology, and policy to formulate an objective view on economically viable energy materials research directions.

The inaugural summit was held in Lisbon, Portugal on October 4-5, 2007, coinciding with the Portuguese Presidency of the European Union. The scope included reduced $\mathrm{CO}_{2}$ emissions during energy production and use by means of new and innovative materials and processes, solar energy, wind power, biofuels, hydrogen fuel cells, nuclear fission and fusion, and complementary problems in storage, transmission, conservation, and waste utilization. Current projections estimate that energy needs of the world will more than double by the year 2050, a demand that cannot be met by existing technologies. In this context, advanced materials, materials research, and innovation are arguably the most urgent elements to prepare new technologies needed in the not-too-distant future.

At the end of the First Summit, the most important aspects identified were published in the "Lisbon 2007 Declaration on International Cooperation in Materials Research: Key to Meeting Energy Needs and Addressing Climate Change." The meeting concluded that the present pace of research and development was too slow to meet the needs of the world's rapidly growing and increasingly energy-hungry population. Effort was too fragmented and competition between different laboratories and institutions - not to mention governments-hindered progress on complex problems where international cooperation is vital to achieve breakthroughs. It was conveyed that each of the technologies discussed required new and improved materials to increase efficiency and reliability, decrease greenhouse gas emissions, reduce capital costs, and extend operating lifetimes. These conclusions were sometimes in conflict with the views expressed by mainstream media. It was recognized, for example, that energy coming from fossil fuels would continue to dominate the energy landscape for the next several decades, hydrogen fuelled vehicles are far from readiness for the mass market at economically valid conditions, and electric automobiles would be limited to small niche markets by Li-battery stability. Moreover, the availability of critical, rare elements for new technologies to meet global demand is a major concern. It was agreed that solar; further controlled use of nuclear; and in the long term, biological energy sources are eligible technologies in which materials research could bring substantial gains in sustainability attributes. The First Summit was addressed by dignitaries from the European Commission, the European Science Foundation, and prominent scientists from industry as well as government laboratories and academia.

The positive outcome from the First Summit immediately led to planning the 